\title{
Les limites du « remodelage socialiste »: les Égyptiens formés en RDA (1969-1989)
}

The Limits to "Socialist Remodeling": Training Egyptians in GDR (1969-1989)

Los límites de la «remodelación socialista»: los egipcios formados en la RDA

(1969-1989)

Amélie Regnauld

\section{(2) OpenEdition}

Journals

Édition électronique

URL : https://journals.openedition.org/remi/7787

DOI : 10.4000/remi. 7787

ISSN : $1777-5418$

Éditeur

Université de Poitiers

Édition imprimée

Date de publication : 1 septembre 2016

Pagination : $57-76$

ISBN : 979-10-90426-27-6

ISSN : 0765-0752

Référence électronique

Amélie Regnauld, «Les limites du « remodelage socialiste » : les Égyptiens formés en RDA

(1969-1989) », Revue européenne des migrations internationales [En ligne], vol. 32 - n² | 2016, mis en

ligne le 01 septembre 2018, consulté le 15 avril 2022. URL : http://journals.openedition.org/remi/7787 ; DOI : https://doi.org/10.4000/remi.7787 


\section{Les limites du " remodelage socialiste ": les Égyptiens formés en RDA (1969-1989)}

\section{Amélie Regnauld ${ }^{1}$}

Le 10 juillet 1969, le gouvernement égyptien rend officielle la reconnaissance de la République Démocratique Allemande (RDA), dans le cadre de la "vague de reconnaissances ${ }^{2}$ dont Berlin-Est fait l'objet par les pays arabes. La mise en place des relations diplomatiques bilatérales parachève le processus de rapprochement économique et culturel entamé par l'Égypte et la RDA depuis le milieu des années 1950. Le 27 février 1965, lors de sa visite en Égypte, le président du Conseil d'État de la RDA, Walter Ulbricht, a en effet déjà inauguré le centre culturel est-allemand du Caire (Wissa-Wassef, 1972 : 626). Le $1^{\text {er }}$ mars 1965, un accord bilatéral a donné lieu à la signature d'une série de conventions visant à organiser les relations culturelles et technico-scientifiques ${ }^{3}$. Depuis, une centaine de places est réservée chaque année aux étudiants et aux travailleurs égyptiens en RDA, au titre de la formation populaire (Volksbildung) ${ }^{4}$. L'encadrement très strict de ces mobilités mène à la constitution de réseaux relationnels étoffés, qui mettent en contact scientifiques, intellectuels et activistes de gauche des deux pays.

Pourtant, de tels liens nourrissent des attentes divergentes : du point de vue est-allemand, I'objectif explicite est de recruter, parmi les étudiants étrangers, les meilleurs ambassadeurs de la RDA ; ces derniers doivent devenir, " une fois leurs études terminées, des activistes expérimentés du mouvement d'amitié

1 Professeur du second degré, agrégée d'histoire, docteure en histoire contemporaine, Collège Adolphe Chérioux, 247 rue Édouard Tremblay, 94400 Vitry-sur-Seine ; amelieregnauld@gmail.com

2 D'après le terme allemand " Anerkennungswelle ".

3 Ministerium für Auswärtige Angelegenheiten der DDR (MfAA), L 187, C 928/77:

Protokollabteilung. Akkreditierung und Abberufung des ägyptischen Botschafters, Saad Badawi al Fatatry, in der DDR. Juli 1969, Dez. 1971. Überblick über die Entwicklung der Beziehungen zwischen der DDR und der VAR.

4 Les plans de travail culturel du MfAA, les rapports de la Stasi et du FDGB permettent dé reconstituer partiellement les flux d'étudiants, de scientifiques et de travailleurs entre I'Égypte et la RDA. 
dans leurs propres pays $»^{5}$. Le comportement et la " morale ${ }^{6}$ des Égyptiens sont étroitement surveillés par les agents de la Stasi et leur formation s'accompagne d'un encadrement idéologique supposé les convertir aux vertus du marxismeléninisme. Du point de vue égyptien, le séjour en RDA répond davantage à la volonté de profiter d'un savoir-faire technique et matériel : il s'insère dans des stratégies de formation individuelles et s'apparente parfois à une étape censée faciliter l'émigration dans un pays occidental.

Le processus de familiarisation réciproque permis par l'immersion linguistique et culturelle est cependant un facteur de rapprochement des élites politiques ou artistiques, même si ces liens n'excluent pas une imbrication d'intérêts, qui ne relèvent pas tous de l'idéologie.

À partir des sources de l'ancienne Allemagne de l'Est (ministère des Affaires étrangères, $\mathrm{SED}^{7}$, Stasi, archives militaires), de documents issus des organisations égyptiennes de gauche (Parti communiste égyptien ou PCE, Tagammu) conservés à I'International Institute of Social History d'Amsterdam, et de la presse est-allemande et égyptienne, cet article se propose d'étudier les ressorts de la coopération culturelle et technico-scientifique égypto-est-allemande et entend montrer les limites de l'entreprise de "remodelage socialiste ${ }^{8}$ " menée par Berlin-Est auprès de ses interlocuteurs égyptiens.

Ce travail s'inscrit dans le cadre d'un double renouvellement historiographique, permis par l'ouverture progressive des archives des anciens pays du bloc de l'Est après 1989 : celui des études sur la politique étrangère des démocraties populaires, et plus particulièrement de la RDA, et celui des recherches transnationales sur le bloc de l'Est, nées dans un contexte " post-guerre froide" (Kott et Faure, $2011: 2$ ) et qui s'intéressent aux " transferts culturels " ${ }^{9}$ (Marès, 2007 : 12), notamment dans le cadre des relations Est-Sud.

5 SAPMO-BArch, DY 13/2349, Liga für Völkerfreundschaft. Büro des Generalsekretärs. Ägypten, 1975, Sekretär Brasch, 27 novembre 1975, Décision du Secrétariat de la Ligue pour l'amitié entre les peuples de RDA sur le travail avec les étrangers étudiant en RDA et avec les diplômés est-allemands dans les établissements scolaires de RDA, le 31 octobre 1975.

6 MfAA, L 187, C 18/76, Abt. Kulturelle Auslandsbeziehungen. Kulturplanungsunterlagen zu den kulturellen Beziehungen zwischen der DDR und Ägypten. 1975-1977. Lettre de Horst Winter, premier secrétaire de I'ambassade de RDA au Caire, au Haut département des relations internationales du ministère de I'Enseignement supérieur et spécialisé, 22 novembre 1976.

7 Sozialistische Einheitspartei Deutschlands (Parti socialiste unifié d'Allemagne).

8 Pour reprendre l'expression d'une analyse est-allemande de 1972, qui estime que " bien que les conceptions égyptiennes du socialisme se différencient de celles des pays socialistes ", l'Égypte " emprunte la voie du remodelage socialiste (sozialistische Umgestaltung) de la société ". Voir : MfAA, L 187, C 767/75, Ministerbüro Winzer. Konsultation zwischen dem Minister für Auswärtige Angelegenheiten der DDR, Otto Winzer, und dem Aussenminister Ägyptens, Dr. Mohamed Hassan Zayyat, zur Nahostproblematik. Okt., Nov. 1972. Information vom 19.10.1972 über die Lage in der ARÄ, Telegramm vom 19. Oktober 1972.

9 En ce qui concerne les démocraties populaires, ce chantier est encore très récent. II faut toutefois rappeler qu'Espagne et Werner (1988) avaient déjà théorisé la notion de transfert culturel, dans le sillage des recherches historiques portant sur les relations entre culture et relations internationales. 


\section{Coopération culturelle et technico-scientifique : la mise en cuvre de la "formation populaire "}

\section{La formation des Égyptiens en RDA : conditions d'éligibilité et essai de quantification}

Les migrations sont strictement encadrées, car le séjour des étrangers en RDA est étroitement réglementé. Comme chaque pays partenaire de Berlin-Est, l'Égypte se voit attribuer chaque année un nombre précis de bourses d'études destinées à des étudiants, des scientifiques ou des travailleurs égyptiens ${ }^{10}$; une centaine de places en moyenne ${ }^{11}$. Ces dernières sont rarement pourvues dans leur intégralité, ce qui traduit d'emblée les limites de l'attractivité de la RDA.

Le programme, la durée et les conditions de la formation sont définis à

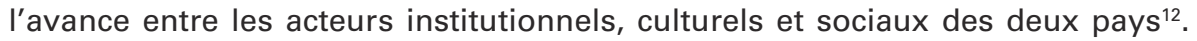
Des critères familiaux, académiques et linguistiques stricts président à l'envoi d'étudiants et de travailleurs égyptiens en RDA.

La quasi-totalité des séjours concerne des hommes, qui doivent séjourner en RDA sans leur famille, témoigner d'un cursus préalable et posséder des connaissances minimales en langue allemande. La plupart du temps, ces dernières sont dispensées par les centres culturels est-allemands du Caire et d'Alexandrie, avant le séjour ${ }^{13}$. De ce point de vue, la sélection des étudiants et travailleurs égyptiens est semblable à celle des autres étrangers : les Vietnamiens par exemple doivent suivre 200 heures de cours d'allemand avant de pouvoir participer à une formation spécialisée (Krüger-Potratz, 1991 : 175). Une fois en RDA pour débuter leur formation, les stagiaires égyptiens sont censés fournir un certificat attestant de leurs connaissances en langue allemande ${ }^{14}$. En réalité, cette règle $n^{\prime}$ est pas strictement appliquée puisqu'elle est régulièrement rappelée ${ }^{15}$. En effet, malgré

10 Les plans de travail culturel du MfAA, les rapports de la Stasi et du FDGB permettent de reconstituer partiellement les flux d'étudiants, de scientifiques et de travailleurs entre I'Égypte et la RDA.

11 En 1971, 3000 étrangers sont recensés en RDA, sur une population totale d'environ 16 millions d'habitants. Ils sont 4200 en 1974, 11650 en 1979 et entre 100000 et 200000 en 1989. Le nombre maximal d'Égyptiens présents en RDA et affiché par les autorités estallemandes est de 600 au début des années 1970.

12 II s'agit des miniștères liés aux domaines concernés par la coopération (notamment des ministères de l'Éducation et de l'Enseignement supérieur, mais aussi de l'Industrie ou de I'Agriculture) et des ligues d'amitié, des comités de solidarité et des syndicats. La Ligue pour I'amitié entre les peuples coordonne par exemple le recrutement direct des étrangers désireux de venir étudier en RDA. L'EFL (Egyptian Federation of Labour), I'alliance des syndicats égyptiens, encadre quant à lui l'envoi d'ouvriers égyptiens pour une formation spécialisée dans une entreprise ou un établissement d'enseignement professionnel de RDA.

13 MfAA, L 187, C 1354/76, Abt. Naher u. Mittlerer Osten. Sektor Ägypten, Sudan, Libyen. Verhandlungen, Abschluss und Erfüllung von Kulturarbeitsplänen zwischen der DDR und der VAR bzw. Ägypten. 1967-1969, 1971, 1973. Arbeitsplan zwischen der Regierung der DDR und der ägyptischen Regierung über die kulturelle und wissenschaftliche Zusammenarbeit in den Jahren 1973 und 1974, Artikel 4.

14 BStU, MfS, Archiv der Zentralstelle, HA XIX 4639, stagiaires de RAE, mai 1984, renvoi de documents concernant d'anciens stagiaires de RAE.

15 MfAA, L 187/C 1902, Abt. Kulturelle Auslandsbeziehungen. Vorbereitung und Abschluss des Kulturarbeitsplanes für 1979/80 zwischen der DDR und Ägypten. 1978-1979.

Stellungnahme zum Kulturarbeitsplanentwurf DDR-Arabische Republik Ägypten für die Jahre 1979 und 1980, Teil Hochschulwesen. 
ces contraintes, le niveau de langue des étudiants égyptiens satisfait peu les formateurs est-allemands ${ }^{16}$. Ponctuellement, I'ambassade de la RDA au Caire conseille d'ailleurs aux institutions scientifiques est-allemandes d'assouplir leurs exigences afin de ne pas envenimer les relations bilatérales ${ }^{17}$.

Les Égyptiens formés en RDA reçoivent une indemnité mensuelle qui est revalorisée d'année en année et qui varie entre 300 et 950 marks est-allemands selon le niveau de qualification de l'individu concerné (Hübner, $1998: 50)^{18}$.

Enfin, les Égyptiens, comme les Vietnamiens ou les Algériens, sont hébergés collectivement, soit en internat, soit dans les logements des entreprises ${ }^{19}$. Leur prise en charge par le régime est-allemand s'inscrit dans la continuité de la stratégie de séduction menée par les États socialistes auprès du tiers-monde depuis le début des années 1960 (Coumel, $2001: 53-71)^{20}$ : il s'agit de forger I'image d'un pays accueillant, généreux et solidaire. En réalité cependant, " les proclamations d'internationalisme participent [davantage] de l'autoreprésentation du régime " (Kott, Lattard et Vincent, $2011: 86)$ que de la solidarité prolétarienne, la prise en charge matérielle des migrants étant souvent le meilleur moyen de les contrôler. Le développement récent des travaux consacrés aux étrangers en RDA (Krüger-Potratz, 1991 ; Behrends, Lindenberger et Poutrus, 2003), et notamment aux populations vietnamienne (Dennis et Weiss, 2005), cubaine (Cala-Fuentes, 2007) ou africaines (Möller, 2001 ; Dagne, 2004 ; Taubert, 2010) met en lumière la contradiction entre "le discours politique de solidarité et la réalité du rejet " (Kott, Lattard et Vincent, $2011: 86)$. Si les sources font peu état des revendications émises par les Égyptiens à propos de leurs conditions de logement, on peut supposer qu'ils sont confrontés aux mêmes problématiques que leurs homologues asiatiques ou africains venus étudier ou travailler en RDA $^{21}$. C'est ainsi qu'en avril 1975, les Égyptiens formés à l'École de technique

16 BStU, MfS, Archiv der Zentralstelle, HA XIX 4639, stagiaires de RAE, mai 1984, renvoi de documents concernant d'anciens stagiaires de RAE.

17 SAPMO-BArch, DY 30/IV B 2/9.04/66, Abteilung Wissenschaften. Bd. 11: Ägypten von 1973, 1975. Rapport (partie 1) sur les négociations entre l'Académie des sciences de RDA et l'Académie des sciences de RAE, pour la période du 20 janvier au 12 février 1973, au Caire.

18 À titre de comparaison, en 1990, le salaire mensuel net moyen en Allemagne de l'Est est de 1131 marks. Il s'agit cependant du salaire net moyen des hommes. À la même date, le salaire net moyen des femmes est de 825 marks.

19 MfAA, L 187, C 1449/78, Abt. Naher u. Mittlerer Osten. Sektor Ägypten, Sudan, Libyen. Arbeitsplan zwischen der Regierung der DDR und der ägyptischen Regierung über die kulturelle und wissenschaftliche Zusammenarbeit in den Jahren 1975 und 1976 und Protokoll über die 3. Tagung des Kulturkomittezs DDR-Ägypten 1976. Juni 1975, März 1976. Arbeitsplan zwischen der Regierung der Deutschen Demokratischen Republik und der Regierung der Arabischen Republik Ägypten über die kulturelle und wissenschaftliche Zusammenarbeit in den Jahren 1975 und 1976 - Artikel 64.

20 L'établissement emblématique des liens privilégiés entre les États socialistes et les pays du tiers-monde est I'Université de l'amitié des peuples de Moscou. Fondée en 1960, cette dernière est spécialement destinée aux étudiants d'Asie, d'Afrique et d'Amérique latine. En mars 1961, elle prend le nom de Patrice Lumumba, ex-Premier ministre du Congo tout juste assassiné.

21 Krüger-Potratz (1991) rappelle ainsi que le confort relatif procuré par l'octroi systématique d'un logement ne doit pas faire oublier les problèmes nés de la promiscuité et de I'éloignement : souvent logés en périphérie des villes, les étrangers sont isolés des Allemands de l'Est, ce qui ne facilite ni leur intégration, ni le bon accueil des habitants. Taubert (2010) évoque de son côté la pénurie de logements que doivent affronter les Algériens ayant migré en RDA. 
agricole de Triptis, en Thuringe, " expriment tous leur insatisfaction en ce qui concerne le logement ${ }^{22}$.

\section{Les "spécialités prioritaires ": encourager les transferts de technologie et favoriser le processus d'industrialisation de I'Égypte}

La coopération bilatérale est orientée avant tout de façon à encourager les transferts de technologie, le processus d'industrialisation et la mise en valeur des ressources naturelles de l'Égypte. Elle se spécialise notamment dans l'industrie lourde et I'hydro-électricité. Dans le domaine agricole, la recherche fondamentale privilégie avant tout des programmes visant à " réso[udre] les problèmes de ravitaillement " et à " accro[ître] la production agricole " ${ }^{23}$, afin de réduire la dépendance alimentaire de l'Égypte à l'égard de l'étranger. Le Caire insiste sur la nécessité de former des " cadres dirigeants dans le secteur industriel étatique ${ }^{24}$ et valorise d'autant plus le savoir-faire technico-scientifique est-allemand qu'il symbolise le succès d'une politique volontariste initiée par un pouvoir étatique fort.

De leur côté, les observateurs est-allemands décrivent la " profonde crise ${ }^{25}$ dans laquelle se trouvent la science, l'art et la culture en Égypte. En 1988, 50 \% de la population âgée de plus de dix ans ne sait ni lire ni écrire, soit 17,16 millions de personnes, et ce bien que depuis 1952, l'école soit gratuite et obligatoire pour les enfants de six à quinze ans.

Ce bilan accablant des deux côtés contribue à donner relativement tôt une base institutionnelle aux projets culturels et technico-scientifiques communs. Dès janvier 1970, les deux pays signent un protocole commun qui organise l'échange d'experts et de scientifiques entre les établissements scientifiques et les entreprises des deux pays. Entre 1970 et 1973, les plus grandes universités est-allemandes et égyptiennes signent des traités d'amitié bilatéraux, comme le montre le tableau ci-dessous :

\footnotetext{
22 SAPMO-BArch, DY 34/10 542, FDGB, Département des relations internationales, Étudiants et stagiaires d'Égypte, d'Algérie, du Maroc et de Bahreïn. Note sur une conversation avec des stagiaires égyptiens le 9 avril 1975, 15 avril 1975.

23 SAPMO-BArch, DY 30/8259, Abteilung Wissenschaften. Internationale wissenschaftlichtechnische Zusammenarbeit (Ägypten), 1981-85. Rapport sur les résultats du séjour d'une délégation de I'Académie des sciences de RDA au Caire, RAE, Académie des Sciences de RDA, Berlin, 26.1.1981.
}

24 SAPMO-BArch, DY 30/2418, ZK der SED, Büro Erich Honecker. Internationale Beziehungen der SED zu Staaten und Parteien. Beziehungen mit Ägypten 1971-1973-19861989. Conceptions communes sur le développement des relations bilatérales entre la RDA et la RAE à I'occasion de la visite officielle du représentant du Premier ministre et ministre des Affaires étrangères de RAE, le Dr. Ahmed Esmat Abdel Meguid, en RDA, en juin 1986.

25 MfAA, L 187, C 64/99, Abt. Naher u. Mittlerer Osten. Aussenkulturbezeichungen zwischen der DDR u. Âgypten. Enthält u. a.: Vorbereitung des Kulturarbeitsplanes 1979/80. Enthält auch: Kulturpolitische Entwicklung Ägyptens 1978/79. - BRD-Aktivitäten im Kulturbereich Ägyptens 1979. 1979. Analyse der kulturpolitischen Entwicklung in der AR-Ägypten 1978/79, Kulturattaché, Kairo, den 31.10.79. 
Tableau 1 : Conventions bilatérales entre les grands établissements d'enseignement supérieur est-allemands et égyptiens

\begin{tabular}{lc}
\hline $\begin{array}{l}\text { Date de la signature } \\
\text { de l'accord de } \\
\text { coopération bilatérale }\end{array}$ & Établissements d'enseignement supérieur concernés \\
\hline $\begin{array}{l}\text { Janvier 1970 } \\
\text { Janvier 1970 }\end{array}$ & $\begin{array}{r}\text { Université Humboldt de Berlin - Université du Caire } \\
\text { Université Karl Marx de Leipzig - Université Aïn } \\
\text { Shams du Caire }\end{array}$ \\
Janvier 1970 & $\begin{array}{r}\text { Institut d'interprètes de l'Université Karl Marx de Leipzig - } \\
\text { École supérieure des langues de Zeïtoun }\end{array}$ \\
1973 & $\begin{array}{r}\text { Université Wilhelm Pieck de Rostock - Université d'Alexandrie } \\
1973\end{array}$ \\
Université Martin Luther de Halle - Université el-Azhar
\end{tabular}

Source : Plans de travail culturel du MfAA.

Les Égyptiens qui viennent étudier ou travailler en RDA se répartissent entre les établissements d'enseignement supérieur, les instituts spécialisés et les entreprises est-allemandes. La majorité d'entre eux suit une formation en sciences de l'ingénieur, en économie ou en technique agricole. Cette spécialisation correspond à l'essor des filières techniques dans tout le tiers-monde, après la décolonisation : les systèmes éducatifs des jeunes États indépendants doivent former des ingénieurs au service du développement national. Véritables " agents techniques du progrès économique ", les ingénieurs, " "enfants gâtés" des nouveaux États "(EI Kenz, 1995 : 568-569), sont encouragés à suivre des formations techniques et scientifiques à l'étranger. Entre 1965 et 1977, 1194 stagiaires égyptiens ont ainsi suivi une formation en RDA, allant de quelques semaines à plusieurs années, dans l'électronique, I'agriculture, la construction de machines ou encore l'industrie textile ${ }^{26}$. Le plus souvent, cette formation a lieu au sein des universités ou des établissements spécialisés ${ }^{27}$.

Plus ponctuellement, la RDA propose aussi des bourses d'études à des médecins, sportifs et artistes égyptiens, pour des missions et des projets spécifiques. Tous les ans, la DHfK (Deutsche Hochschule für Körperkultur) de Leipzig propose par exemple des formations spécialisées de huit à dix mois en sports de combat, natation ou pédagogie du sport, à deux ou trois entraîneurs sportifs

26 BStU, MfS, Archiv der Zentralstelle, SdM 2495, 1978, Rapport sur I'état des relations économiques, culturelles et scientifiques entre la RDA et la RAE.

27 À la fin de l'année 1977 par exemple, la Stasi recense soixante-douze Égyptiens en formation en RDA : parmi eux, soixante sont inscrits dans les différents instituts de recherche de l'Académie des Sciences. Les douze individus restants travaillent quant à eux dans des entreprises propriétés du peuple (Volkseigene Betriebe) dispersées dans le pays, à Dresde, Gera ou Berlin. L'Académie des Sciences regroupe plusieurs instituts de recherches disséminés dans le pays : I'Institut pour la biochimie des plantes de Halle, I'Institut pour la chimie de Teltow, le Centre de recherches de Rossendorf, I'Institut central pour la physique de la terre de Potsdam, I'Institut central pour la physique des matériaux et les matières premières de Dresde et I'Institut central d'histoire. Voir BStU, Zentralarchiv, MfS, HA XVIII 6855, Berlin, 8 décembre 1977, Situation des citoyens de RAE qui se trouvent en RDA à long terme, dans le cadre de conventions étatiques. 
égyptiens ${ }^{28}$. Ces derniers sont diplômés en RDA et obtiennent le grade académique de Bachelor of Arts $^{29}$. De retour en Égypte, ils peuvent valoriser cette qualification supplémentaire : en 1969 par exemple, l'entraîneur de l'équipe nationale de football d'Égypte, Mohamed Abou Saleh, est un ancien étudiant de la $D H f K$ de Leipzig, dont il est sorti diplômé dix ans auparavant ${ }^{30}$.

\section{Formation technique ou idéologique?}

\section{Pour la RDA : former des " activistes expérimentés " ${ }^{31}$ et de futurs partenaires. Les objectifs extra-scientifiques de la coopération culturelle}

Du point de vue est-allemand, l'objectif explicite qui guide la coopération culturelle et technico-scientifique est de faire des étudiants étrangers, après leur retour dans leur pays d'origine, des meneurs "progressistes ", " en situation de diriger un collectif [...] $\|^{32}$. Comme le constate Taubert lorsqu'il analyse le processus de formation des ouvriers algériens en RDA au cours des années 1960, Berlin-Est caresse I'espoir, en renvoyant des travailleurs qualifiés dans leurs pays, "d'y importer des idées bien spécifiques d'organisation sociale " (Taubert, $2010:$ 143).

Certains instituts sont spécialisés dans l'accueil des étrangers. C'est le cas de I'Institut Herder de I'Université Karl Marx de Leipzig, qui dispense aux étudiants étrangers des cours d'éducation politique. L'expérience prouve que la stratégie pragmatique de la RDA porte ses fruits, dans une certaine mesure : la coopération culturelle est un moyen relativement efficace de nouer des relations durables avec les élites égyptiennes, et notamment avec les personnalités politiques de gauche. Si l'offensive culturelle est-allemande en Égypte ne touche guère les masses, la plupart des interlocuteurs égyptiens privilégiés de Berlin-Est a en revanche effectué une partie de sa formation en RDA ou

\footnotetext{
28 MfAA, L 187, C 1449/78, op.cit., Arbeitsplan zwischen der Regierung der Deutschen Demokratischen Republik und der Regierung der Arabischen Republik Ägypten über die kulturelle und wissenschaftliche Zusammenarbeit in den Jahren 1975 und 1976 Artikel 53.

29 MfAA, L 187, C 18/76, Abt. Kulturelle Auslandsbeziehungen. Kulturplanungsunterlagen zu den kulturellen Beziehungen zwischen der DDR und Ägypten. 1975-1977. Offres de places à la $D H f K, 29$ juin 1976.

30 Neues Deutschland, 10 juillet 1969, "L'échange amical RDA-RAU s'est achevé 7-0 ", p. 8.

31 SAPMO-BArch, DY 13/2349, op.cit., 27 novembre 1975, Décision du Secrétariat de la Ligue pour l'amitié entre les peuples de RDA sur le travail avec les étrangers étudiant en RDA et avec les diplômés est-allemands dans les établissements scolaires de RDA, le 31 octobre 1975.

32 SAPMO-BArch, DY 34/10 542, op.cit. Dossiers de stagiaires.
} 
dans un État socialiste, ou y a séjourné ponctuellement ${ }^{33}$. En Égypte, les partenaires politiques de Berlin-Est, qu'ils soient membres du gouvernement ou qu'ils appartiennent à des organisations paraétatiques ont en effet souvent une connaissance minimale de la RDA. Le développement de liens particuliers avec les fonctionnaires de l'Institut national de la planification ${ }^{34}$ du Caire constitue un exemple significatif de cette stratégie $d^{\prime}$ " infiltration " politique, par le biais de réseaux personnels savamment entretenus depuis des mois, parfois des années. En coopération avec les grandes institutions internationales, comme la Banque mondiale, l'Institut évalue les programmes et les projets d'investissement et de développement en Égypte, menés dans les secteurs économiques et sociaux. En 1978, environ $70 \%$ des cinquante-cinq collaborateurs scientifiques de I'Institut " ont reçu le grade de docteur dans les États socialistes " ${ }^{35}$. En 1983, I'Institut est encore "un haut lieu des diplômés de RDA " ${ }^{36}$. Du point de vue est-allemand, le recrutement sociologique de I'Institut national de la planification est un véritable atout diplomatique, car " les Égyptiens diplômés en RDA jouent [ainsi] un rôle significatif dans l'économie égyptienne $\|^{37}$. Inversement, les acteurs égyptiens utilisent ces réseaux de connaissances pour mener à bien certains de leurs projets.

Pour Berlin-Est, I'Institut national de la planification fait figure de réservoir de personnalités progressistes, susceptibles de relayer les conceptions estallemandes en matière d'organisation socio-politique. Cette proximité remonte à 1969, date de la nomination au poste de directeur d'Ismaïl Sabri Abdallah, économiste d'inspiration marxiste ${ }^{38}$. En 1968-1969, ce dernier a voyagé en URSS puis noué des liens avec l'École supérieure d'Économie de RDA et le Comité

33 C'est le cas de Rifaat el-Saïd, journaliste né en 1932, membre du PCE clandestin et du parti de gauche légal, créé en 1976, le PRNPU ou Tagammu. Ayant séjourné en RDA dans la première moitié des années 1970, ce dernier est diplômé de I'Université Karl-Marx de Leipzig, où il a soutenu un mémoire de maîtrise à l'Institut du marxisme-léninisme sur "le mouvement socialiste de RAE, de 1900 à 1925 ". II a ensuite entamé une thèse de doctorat en histoire, à Leipzig, tout en poursuivant ses activités politiques en Égypte. Ce cursus lui a permis de nouer des liens avec des hauts fonctionnaires est-allemands et de jouer le rôle d'intermédiaire entre les organisations de gauche en Égypte et le régime de Berlin-Est. En RDA, Rifaat el-Saïd se trouve ainsi au croisement de plusieurs circuits relationnels, ce qui lui permet de concilier objectifs politiques et ambitions professionnelles individuelles.

34 Fondé en 1960, I'Institut national de la Planification est dirigé par un comité dont le président est également le ministre de la Planification. L'organisme coordonne des études qui lui ont été commandées par les organes d'État égyptiens. Voir MfAA, L 187, C 64/64, Abt. Naher u. Mittlerer Osten. Informationen über Gespräche von DDR-Diplomaten und Vertretern des Solidaritätskomitees der DDR mit ägyptischen Persönlichkeiten nach oder bei Besuchen im Gastland, 1978, Vermerk über den Besuch des Instituts für Nationale Planung der ARÄ, Botschaft der DDR in der AR Ägypten, Kairo, 23.4.78.

35 Ibid.

36 SAPMO-BArch, DY 30/13 667, Abteilung Internationale Verbindungen im ZK der SED. Ägypten. Bd. 1: 1981-1986. Vermerk über ein Gespräch des Präsidents des Sekretariats des Nationalrats der Nationalen Front der DDR, Werner Kirchhoff, mit dem Generalsekretär der VNPP Ägyptens, Dr. Rifaat el-Saïd, den 7.4.1983, Abt. Internationale Verbindungen, Berlin, 11.4.1983.

37 Ibid.

38 MfAA, L 187, C 641/73, Abt. Arabische Staaten/ Ägypten. Vermerke über Gespräche mit Vertretern Ägyptens in Ägypten. 1968-1970. Vermerk über ein Gespräch mit dem neuernannten Direktor des Nationalen Planungsinstituts der VAR, Dr. Ismail-Sabri Abdalla am 2.9.1969, Botschaft der DDR in der VAR, Kairo, den 2.9.1969. 
central du SED. En janvier 1972, il devient lui-même ministre de la Planification : à ce titre, il est l'un des rares membres du gouvernement égyptien à être considéré " comme un ami par la RDA " $^{39}$.

Ce sont véritablement ces réseaux relationnels étoffés par la coopération culturelle bilatérale qui offrent à la RDA ses meilleurs interlocuteurs politiques en Égypte. Ponctuellement, Berlin-Est parvient ainsi à nouer des contacts durables avec certaines personnalités influentes dans le paysage politique égyptien. En revanche, l'impact de son offensive culturelle sur la majorité de la population égyptienne est beaucoup plus restreint.

\section{Pour l'Égypte : profiter d'un savoir-faire technique et de facilités matérielles. Les limites de la formation idéologique}

$C^{\prime}$ est le constat que font régulièrement les acteurs est-allemands confrontés au terrain égyptien : ils admettent que le " remodelage socialiste " des personnes qui séjournent en RDA reste bien souvent une gageure. D'ailleurs, si Le Caire encourage la formation de ses ressortissants en RDA, c'est aussi parce que ces derniers " [rentrent] toujours en Égypte " et ne sont guère tentés de $s^{\prime}$ établir durablement dans la République socialiste ${ }^{40}$.

En mars 1976, Ahmed Maher Mohamed, stagiaire à la VEB Bi Binnenhäfen de Magdeburg se montre " très intéressé " par sa formation, du moins tant qu'elle répond à ses besoins ${ }^{42}$. II n'est visiblement pas très sensible aux questions ayant trait au mode de direction des entreprises, tout simplement parce qu'il estime que les structures entrepreneuriales " sont différentes en Égypte ". Enfin, selon ses évaluateurs, "il ne comprend pas trop le socialisme " et ne se montre pas particulièrement curieux en la matière. Les différents informateurs soulignent tous son indifférence pour le modèle politique est-allemand : " ses informations sur la RDA sont sommaires " et " il ne connaît pas les mesures prises " dans le pays $^{43}$.

De plus, les étudiants et travailleurs égyptiens présents en RDA se trouvent parfois à la croisée de plusieurs systèmes d'allégeance politique, qui sont autant de contrepoids à l'encadrement idéologique est-allemand. Le gouvernement égyptien, tout comme les militants de gauche expatriés en Europe, entendent sinon contrôler, du moins influencer cette population émigrée. L'ambassadeur égyptien à Berlin-Est conserve un lien minimal avec ses concitoyens dans le

39 SAPMO-BArch, DY 30/IV B 2-20 430, Abteilung Internationale Verbindungen im ZK der SED. Aussenpolitik der DDR und Beziehungen mit Staaten. Bd. 1: 1972-1973. Lettre de Fritz Weisshuhn au directeur du département des relations internationales du Comité central du SED, Paul Markowski, Berlin, 7.8.1972.

40 MffA, L 187 C 65/00, Abt. Naher u. Mittlerer Osten. Beziehungen zwischen der DDR und Ägypten auf dem Gebiet des Hochschulwesens. 1978-1979. Information über ein Treffen mit Partern des Hochschulministeriums der ARÄ, Kulturattaché, Kairo, den 28.11.79.

41 Volkseigener Betrieb (entreprise propriété du peuple).

42 BStU, MfS, Archiv der Zentralstelle, HA XIX 4639, stagiaires de RAE, mai 1984, renvoi de documents concernant d'anciens stagiaires de RAE, "Évaluation de I'activité du citoyen égyptien Ahmed Maher Mohamed, stagiaire à la VEB Binnenhäfen ", Magdeburg, 11 mai 1976.

43 Ibid., 29 avril 1976, rapport de Willi Werrang. 
pays : dans les années 1970, il s'adresse par exemple deux fois par an aux étudiants égyptiens de Halle ${ }^{44}$. Selon lui, il est de toute façon évident que les ressortissants égyptiens de RDA soutiennent sans faillir leur gouvernement, même lorsque celui-ci mène une politique contraire aux intérêts de leur pays d'accueil $^{45}$ : c'est ce qu'il avance par exemple en décembre 1977, lors de la décision unilatérale de Sadate de fermer les centres culturels est-allemands en Égypte. Pourtant, certains Égyptiens de RDA sont vraisemblablement en contact avec des groupes politiques, parfois clandestins, souvent hostiles à leur régime. Ces derniers tentent en effet de constituer des réseaux d'activistes à l'étranger, parmi les cercles d'étudiants expatriés. Après les arrestations massives d'opposants politiques lors des émeutes de janvier 1977 en Égypte, le parti de gauche autorisé, le Parti du rassemblement national progressiste unioniste (PRNPU) ${ }^{46}$, invite I'Union des étudiants égyptiens à prendre position contre la répression ${ }^{47}$. Le parti coopère avec certaines associations étudiantes d'obédience nassérienne, comme le Club de la pensée socialiste, dont certains membres ont été incarcérés en janvier $1977^{48}$. En 1986, dans la même volonté de ralliement des associations étudiantes à l'opposition de gauche, des membres du Parti communiste égyptien se rendent auprès des étudiants égyptiens de Slovaquie, afin de savoir comment se passe leur vie quotidienne et de connaître leurs éventuelles "requêtes ${ }^{49}$.

En fait, au-delà des sollicitations politiques diverses dont les Égyptiens de RDA peuvent faire l'objet, leur désintérêt relatif pour la formation idéologique qui leur est dispensée s'explique par une raison extrêmement pragmatique : ces derniers viennent dans le pays pour profiter du savoir-faire technico-scientifique est-allemand et des facilités matérielles mises en œuvre afin d'encourager leur migration. Si l'impact de l'action culturelle est-allemande reste relativement limité en termes de pénétration idéologique, la mise en relation des populations contribue pourtant à produire des interactions culturelles, dont les effets peuvent revêtir un caractère politique.

44 MfAA, L 187, C 1430/78, Abt. Naher u. Mittlerer Osten. Sektor Ägypten, Sudan, Libyen. Vermerke über Gespräche von Vertretern des Aussenministeriums der DDR mit ägyptischen Diplomaten. 1975-1977. Vermerk über ein Gespräch des Stellvertreters des Ministers für Auswärtige Angelegenheiten der DDR, Gen. Dr. Willerding, mit dem Boschafter der AR Ägypten in der DDR, Mustafa Mohamed Tewfik, am 26.8.1970, von 10.15 Uhr bis 11.10 Uhr.

45 BStU, MfS, HA XX, AKG, Nr. 6680, Information. Opinions de diplomates égyptiens en RDA, HA XX, strictement confidentiel, Berlin, 22 décembre 1977.

46 Le nom usuel du Parti du Rassemblement National Progressiste Unioniste est le Tagammu.

47 IISH, Michel Kamel Papers, Human Rights, N²18: Reports on the trials of political detainees of the uprising of 1977, in French. Appel de Khaled Mohieddine, secrétaire du PRNPU, le Caire, 3 octobre 1977.

48 IISH, Michel Kamel Papers, Human Rights, N²19: News reports on human rights in Egypt, in French, 1977. Extrait d'un article du journal Le Monde, daté du 25 janvier 1977. 49 IISH, Michel Kamel Papers, 2nd general conference of the CPE, N¹27: Report to the foreign section on Egyptian students in Eastern Europe, in Arabic, 1986. 


\section{La " solidarité internationale vécue au quotidien ${ }^{50}$ : interactions culturelles et gestion des imprévus}

\section{La surveillance des Égyptiens en RDA}

Les organes de la Stasi et notamment le département $X$, chargé des relations internationales (Abteilung $X$, Internationale Verbindung), supervisent la surveillance des étudiants et des travailleurs égyptiens présents en RDA. À l'échelle locale, le matériel d'information brut est rassemblé par les administrations de district et par des "source[s] non officielle[s] " ${ }^{51}$, les Inofizielle Mitarbeiter ${ }^{22}$ ou $I M$, dont le nombre total est estimé à 180000 entre 1975 et 1989 (Combe, $2005: 54-57)^{53}$. II arrive que certains formateurs fournissent des rapports sur leurs propres stagiaires, comme c'est le cas en 1976 au sein de l'entreprise VEB Binnenhäfen de Magdeburg ${ }^{54}$. Vraisemblablement élaborés à partir de conversations informelles avec le stagiaire, ils abordent des questions disparates, souvent sans lien avec la formation professionnelle : les préférences alimentaires, la pratique religieuse, la situation familiale et la vie personnelle. Les " amis " constituent parfois un autre réservoir utile d'informateurs : après avoir lié connaissance avec des Égyptiens, ils rapportent au ministère de la Stasi les renseignements qu'ils ont accumulés. En 1977-1978, " Inge " s'est visiblement constitué un petit réseau de camarades parmi les étudiants égyptiens de Berlin. Introduite auprès des membres de l'association étudiante Nasser Verband, elle fournit à plusieurs reprises des rapports à la Stasi, dont le contenu informatif est très relatif : on y apprend tantôt que I'un " porte des lunettes, [...] est grand, musclé, très fort ${ }^{55}$, tantôt que l'autre " possède beaucoup d'argent et achète vraisemblablement ses cigarettes à I'Ouest $n^{56}$.

Ponctuellement, les sources est-allemandes font état des " activités subversives ${ }^{57}$ organisées par des ressortissants étrangers en RDA. Le régime est-allemand ne manque pas, d'ailleurs, d'instrumentaliser de telles entreprises, ce qui n'est pas sans effet sur ses relations diplomatiques. En novembre 1977, plusieurs manifestations ont lieu à Berlin-Est, à l'initiative d'étudiants arabes, afin de dénoncer le déplacement de Sadate à Jérusalem. Le 18 novembre, vingt-quatre étudiants arabes se rendent ainsi devant l'ambassade d'Égypte à Berlin pour

50 Moine, $2010: 299$.

$51 \mathrm{BStU}, \mathrm{MfS}$, Archiv der Zentralstelle, Abt. X 483, Rapport d'une source non officielle, 1978.

52 Collaborateur non officiel.

53 Combe (2005) rappelle que selon les statistiques, un Allemand de l'Est sur trois a été amené à collaborer au moins une fois dans sa vie avec la Stasi, trois sur cinq d'après d'autres calculs.

54 Le stagiaire Ahmed Maher Mohamed fait l'objet de plusieurs rapports d'IM transmis à la Stasi, dont au moins un est rédigé par I'un de ses formateurs. Voir BStU, MfS, Archiv der Zentralstelle, HA XIX 4639, stagiaires de RAE, mai 1984, renvoi de documents concernant d'anciens stagiaires de RAE, Magdeburg, 29 avril 1976.

55 BStU, MfS, Archiv der Zentralstelle, HA II 28 103, Rapport de "Inge » au Capitaine Scholz, Berlin, 12 décembre 1977.

56 BStU, MfS, HA II 28 103, Rapport de "Inge ", 20 février 1978.

57 SAPMO-BArch, DY 30/IV B 2-20 344, Abteilung Internationale Verbindungen im ZK der SED. ASU: Informationen über die Politik der ASU, 1972-75. Bd. 1: 72-73. 12.7.1972, Abteilung Auslandsinformation. 
exprimer leur mécontentement ${ }^{58}$. D'après la Stasi, il s'agit surtout d'Irakiens, de Libanais et de Palestiniens venus de Potsdam, Rostock, Dresde et Leipzig. Ces derniers entreprennent de lire une résolution dans laquelle ils condamnent le voyage de Sadate en Israël, puis ils clament en chœur plusieurs slogans : " $A$ bas Sadate ! ", "À bas I'impérialisme ! ". Les sources ne précisent pas si parmi les manifestants se trouvent également des étudiants de nationalité égyptienne. Les diplomates égyptiens en fonction en RDA assurent néanmoins que leurs concitoyens approuvent la politique de Sadate, pour des raisons d'ailleurs parfois plus personnelles qu'idéologiques : " une grande partie des étudiants espère une rupture des relations diplomatiques entre l'Égypte et la RDA. Ils pensent qu'ainsi, ils pourraient aller continuer à étudier en RFA ${ }^{59}$. Pourtant, des sources est-allemandes rapportent les craintes de certains étudiants égyptiens : alors que les membres de l'association Nasser ont adressé une lettre de soutien à Sadate, les doctorants qui ne sont pas affiliés à l'organisme redoutent des mesures de représailles. Craignant d'être rappelés en Égypte pour effectuer leur service militaire, ils se méfient de leurs camarades adhérant à l'association, qui eux " ont de bonnes relations avec le gouvernement égyptien "60. Quoi qu'il en soit, les troubles qui agitent les communautés d'étudiants arabes en RDA agacent fortement le régime égyptien, d'autant que les autorités est-allemandes $\mathrm{n}^{\prime}$ entravent pas l'action des manifestants ${ }^{61}$. Conformément à la ligne du bloc soviétique, Berlin-Est refuse en effet toute négociation séparée avec Israël. En octobre 1980, le ministère égyptien de l'Éducation interdit aux étudiants, aux scientifiques et aux professeurs de se rendre dans les États socialistes ${ }^{62}$. Officiellement, le régime justifie cette décision par la situation tendue qui prévaut en Europe de l'Est, face au large mouvement de contestation sociale initié par Solidarnosc en Pologne. Mais pour Berlin-Est, la véritable raison d'une telle interdiction est liée aux manifestations d'étudiants égyptiens qui se sont déroulées à Budapest au cours de l'année 1980 : ces derniers ont occupé leur ambassade afin d'obtenir une augmentation de leurs bourses, tout en formulant des critiques à l'encontre du régime de Sadate. Si ce type d'initiatives, mêlant revendications sociales et politiques, embarrasse Le Caire, elles sont aussi instrumentalisées ponctuellement par Berlin-Est, qui, tout en les surveillant de

58 BStU, MfS, ZAIG, Nr. 15727, Information n726/77 sur le déroulement d'une manifestation de protestation d'étudiants arabes en RDA, devant I'ambassade de RAE en RDA, strictement confidentiel, Berlin, 18.11.1977.

59 BStU, MfS, HA XX, AKG, Nr. 6680, Information. Opinions de diplomates égyptiens en RDA, HA XX, strictement confidentiel, Berlin, 22 décembre 1977.

60 BStU, MfS, HA II, Nr. 28103, Information, Berlin, 6.12.1977.

61 En novembre 1977, face à la lenteur des pourparlers en vue de la tenue d'une conférence internationale de la paix destinée à régler le conflit au Proche-Orient, Sadate déclare devant l'Assemblée nationale égyptienne qu'il est prêt à se rendre en Israël pour négocier la paix. $C^{\prime}$ est un choc psychologique considérable : la visite s'apparente à une reconnaissance de facto de l'État d'Israël et vaut à Sadate I'hostilité des autres États arabes. En décembre, alors que Sadate se rend à nouveau à Jérusalem, I'Égypte est sommée par ses partenaires socialistes de clarifier sa politique proche-orientale. Le bloc de l'Est s'indigne de cette politique de " capitulation ". Les diplomates est-allemands imputent les négociations séparées menées par Sadate à la pression de la bourgeoisie égyptienne, mécontente, selon eux, de voir le conflit au Proche-Orient peser sur le développement capitaliste du pays. Dès 1978, l’Égypte est bannie par le ministère est-allemand des Affaires étrangères du groupe des quinze États arabes et africains suivant une " orientation socialiste ".

62 BStU, MfS, HA XX, Nr. 3893, Interdiction de sortie pour les étudiants, professeurs et scientifiques de RAE, 22.10.1980. 
près, y voit un moyen de pression autant qu'une légitimation a posteriori de sa propre politique.

Dans certains cas, l'encadrement étroit des ressortissants égyptiens en RDA peut produire de la politisation, les "interactions idéologiques " (Katsakioris, 2006 : 16) favorisant des prises de position et des manifestations à caractère politique, que ce soit contre son pays d'origine ou contre son pays d'adoption.

\section{Mécanismes d'adaptations et confrontations socio-culturelles : entre pragmatisme et préjugés}

Les archives de la Stasi et du Freier Deutscher Gewerkschaftsbund (FDGB) ${ }^{63}$ donnent ponctuellement des indications sur la façon dont se déroule le séjour des Égyptiens en RDA, du moins tel qu'il est perçu et/ou rapporté par les observateurs est-allemands responsables de leur encadrement et de leur surveillance. Si certains documents dressent un bilan tout à fait positif de leur séjour, d'autres néanmoins font état de difficultés entre les stagiaires et leurs formateurs, déplorent le comportement inapproprié des Égyptiens ou véhiculent des préjugés éculés. II convient de prendre garde cependant à l'effet de sources, les documents ne rapportant " naturellement que les "mauvaises nouvelles" " (Taubert, 2010 : 144). Ces derniers donnent à voir malgré tout le ressenti des évaluateurs est-allemands face à d'éventuels décalages de perception ou de possibles altercations.

Les reproches récurrents adressés aux Égyptiens concernent leur indiscipline, leur formation insuffisante et leur manque d'ardeur au travail. Au niveau diplomatique comme au niveau de la société civile, le discours est-allemand reprend souvent l'idée selon laquelle les Égyptiens entendent profiter du système social et des avancées technologiques et industrielles d'un pays plus développé que le leur. Pourtant, la RDA joue sur cette image et contribue elle-même à alimenter la conception instrumentale que les Égyptiens peuvent avoir de leur séjour dans le pays $^{64}$. Berlin-Est entretient son image de pays industrialisé et prospère auprès du tiers-monde, tout en regrettant l'intérêt accru que portent les Égyptiens aux produits de consommation plutôt qu'aux programmes culturels et aux formations idéologiques qui leur sont proposés. En 1976, la Ligue pour l'amitié entre les peuples invite Abd al-Salam al-Zayyat, président de la société d'amitié égypto-soviétique, ainsi que sa sœur, Latifa al-Zayyat, professeur de littérature anglaise, en RDA ${ }^{65}$. Mais les invités refusent de se plier à l'itinéraire qu'on leur présente parce qu'ils préfèrent "faire des achats ", " rencontrer des Égyptiens " et qu'ils ont prévu de passer des examens médicaux dans une clinique pour diplomates. Finalement, "malgré plusieurs tentatives ", ils ne voient guère de Berlin que sa fameuse tour de télévision.

\section{Confédération syndicale de RDA.}

64 Le FDGB prend par exemple en charge les coûts d'empaquetage et de transport des biens de consommation (machines à coudre, robots ménagers, etc.) que les stagiaires égyptiens ont achetés en RDA et qu'ils souhaitent rapporter en Égypte, à hauteur de 200 kilos par personne. Voir SAPMO, FDGB, DY 34/10 542, op.cit. Note sur une conversation avec treize collègues égyptiens à l'École spécialisée en technique agricole, le 12 novembre 1975.

65 MfAA, L 187, C 1440/78, Abt. Naher u. Mițtlerer Osten. Sektor Ägypten, Sudan, Libyen. Delegationsaustausch der DDR mit Âgypten. 1974-1977. Rapport sur la délégation III/16 B- RAE, Berlin, 20 janvier 1977. 
Ce bilan en demi-teinte est révélateur du nécessaire pragmatisme dont les interlocuteurs est-allemands et égyptiens doivent faire preuve au cours de leurs échanges. Nombre d'acteurs est-allemands regrettent le manque d'intérêt des Égyptiens pour leur modèle socio-politique, mais s'en accommodent : ils en concluent que ces derniers " ne sont pas mûrs et [...] peu formés " ${ }^{66}$. Ils pointent le caractère utilitaire de la migration en RDA avec un mélange de réalisme et de stéréotypes, à l'image de "Inge " qui en 1978 résume ainsi la démarche des doctorants égyptiens de Berlin : " ils veulent obtenir le titre de docteur sans faire trop d'efforts [...]. Pour beaucoup, le système capitaliste leur plaît, mais à I'université ils montrent de l'enthousiasme pour le socialisme. Les plus brillants iront terminer leurs études aux États-Unis ou en Grande-Bretagne " ${ }^{67}$.

II arrive que ces décalages socio-culturels mènent à la mise en scène publique et visible d'opinions ou de revendications à caractère politique, ce qui apparaît comme un facteur de désordre potentiel pour les régimes en place ${ }^{68}$. En RDA, le discours tenu par certains stagiaires égyptiens contribue à véhiculer une image relativement négative du pays. Parfois anecdotiques, les critiques qu'ils formulent n'en revêtent pas moins une dimension politique, ne serait-ce que parce qu'elles remettent en cause le postulat selon lequel la vie en RDA est forcément plus agréable que le quotidien dans un pays du tiers-monde. La vision que le stagiaire Ahmed Maher Mohamed a de la République est-allemande, par exemple, est d'autant plus problématique que ce dernier est par ailleurs crédité d'une grande rigueur morale par ses interlocuteurs. II blâme pourtant la qualité des produits de consommation : le riz égyptien est meilleur que celui que I'on trouve en RDA, le coton soviétique est quant à lui de moins bonne qualité que le coton égyptien ${ }^{69}$. Il dit avoir été étonné de constater que la télévision en couleurs existait en Allemagne de l'Est, ce qui ne manque pas de vexer son interlocuteur. De ce point de vue, l'Égyptien ne fait que formuler à haute voix la réalité d'une économie de pénurie, visible même par les citoyens issus de pays du tiersmonde. Enfin, il ne se gêne pas pour déplorer le fait qu'« il y [ait] trop de choses interdites en RDA " et critique notamment le contrôle systématique du courrier ${ }^{70}$.

Au quotidien, la gestion des ressortissants égyptiens n'a vraisemblablement que peu d'impact directement politique. En revanche, des questions d'ordre privé se posent aux autorités publiques, et notamment celles des mariages entre ressortissants nationaux et étrangers. Loin d'être anecdotique, la mixité sociale née de la migration peut contribuer à modifier ou à faire évoluer les "filtres idéologiques " dominants dans le pays ; et de ce point de vue, indirectement, elle revêt un caractère politique. En RDA, les sources à disposition nous permettent d'ailleurs de constater que les demandes de mariages entre hommes

66 BStU, MfS, Archiv der Zentralstelle, HA II 28 103, Rapport de " Inge », 14 novembre 1978. $67 \mathrm{Ibid}$.

68 En novembre 1977 par exemple, plusieurs manifestations ont lieu à Berlin-Est, à l'initiative d'étudiants arabes, afin de dénoncer le déplacement de Sadate à Jérusalem. Voir BStU, MfS, Zentralarchiv, ZAIG 15 727, Information sur le déroulement d'une manifestation de protestation d'étudiants arabes en RDA, devant l'ambassade de RAE en RDA, strictement confidentiel, Berlin, 18 novembre 1977, $n^{\circ} 726 / 77$.

69 BStU, MfS, Archiv der Zentralstelle, MfS, HA XIX 4639, stagiaires de RAE, mai 1984 , renvoi de documents concernant d'anciens stagiaires de RAE, Rapport de Willi Werrang sur Ahmed Maher Mohamed, Magdeburg, 29 avril 1976.

70 Ibid., Rapport final sur le stage d'Ahmed Maher Mohamed, 25 mai 1976. 
égyptiens et femmes est-allemandes font l'objet d'un examen scrupuleux de la part des autorités étatiques. Les sources consultées ne permettent aucunement de dresser un bilan statistique fiable des mariages binationaux conclus entre 1969 et 1989, ni de quantifier précisément le nombre de ressortissants étrangers qui décident finalement de rester dans le pays où ils ont migré. II semble globalement que l'installation durable de travailleurs égyptiens dans les pays socialistes, notamment par le biais du mariage, soit relativement restreinte. D'après les indications délivrées par les sources, la RDA ne semble pas faire figure de destination très attractive, contrairement aux États occidentaux. C'est ce que confirme en tout cas Mohamed Hassanein Heikal ${ }^{71}$ qui estime que sous les gouvernements de Nasser et de Sadate, sur les 200000 Arabes ayant séjourné en Union soviétique, moins de $1 \%$ ont épousé une Soviétique, tandis que sur 15000 étudiants arabes présents aux États-Unis durant la même période, 7000 ont épousé une Américaine (Laurens, 1987 : 60). Les archives du FDGB, du ministère de I'Intérieur et de la Stasi contiennent toutefois un certain nombre de dossiers pour les années 1976, 1977 et 1979, qui donnent des indications ponctuelles tout à fait intéressantes sur les stratégies matrimoniales élaborées par les couples binationaux (telles du moins qu'elles sont présentées par les institutions étatiques), ainsi que sur les réponses administratives qui leur sont apportées.

En 1976-1977, le ministère est-allemand de l'Intérieur comptabilise au moins cinquante-et-une demandes de mariages entre des ressortissantes de RDA et des Égyptiens travaillant ou ayant travaillé en Allemagne orientale ${ }^{72}$. Ces demandes sont déposées auprès des conseils de districts de Rostock, Halle, Erfurt, Gera, Dresde, Karl-Marx Stadt, Leipzig et Berlin. Le ministère de la Sécurité d'État mène alors de petites enquêtes afin d'évaluer si la demande est recevable ou non : sur cinquante-et-une demandes, trente-trois dossiers sont conservés dans les archives, dont dix-sept donnent lieu à une approbation. Les motifs de l'autorisation ou du refus des mariages binationaux sont parfois confus. II semble généralement, néanmoins, que l'autorisation de mariage dépende de la façon dont les organes de sécurité évaluent la conformité du comportement de la demanderesse à une norme socio-politique vaguement prédéfinie : un quotidien " stable ", pas de relations connues avec l'Ouest, une parentèle ne détenant pas de compétences ou d'informations indispensables à la sécurité d'État.

Les dossiers élaborés par la Stasi s'attachent surtout à enquêter sur les mœurs et les opinions des ressortissantes est-allemandes. En revanche, ils ne donnent que peu d'informations sur les citoyens égyptiens eux-mêmes. Pourtant, le refus du mariage peut aussi être motivé par les clauses bilatérales qui règlementent le séjour des travailleurs égyptiens en RDA : officiellement, ces dernières sont en tout cas parfois brandies pour justifier l'interdiction du mariage. En février 1976, Ali Abed el-Cham Abdel Aziz, stagiaire depuis deux ans au sein d'une entreprise de construction d'équipements électroniques, la VEB Starkstrom Anlagenbau, à Erfurt, se présente au département des relations internationales du FDGB ${ }^{73}$. Accompagné de sa fiancée, il sollicite le droit de rester en

71 Journaliste, rédacteur en chef d'al-Ahram en 1957, il devient ministre de I'Information en 1970 sous Nasser, dont il est I'un des conseillers.

72 SAPMO-BArch, DO 1/14 857, Ministerium des Innern. Abt. Bevölkerungspolitik. Bd. 34, Eheschliessungen. 1976/1977-1987. Dossier Égypte.

73 SAPMO-BArch, DY 34/10 542, op.cit. Dossier de Ali Abed el-Cham Abdel Aziz, Berlin. 
RDA à la fin de sa formation, prévue pour juillet 1976, afin de pouvoir épouser cette dernière, avec laquelle il a eu un enfant ${ }^{74}$. II exprime son souhait d'obtenir un contrat de travail dans son entreprise, afin de "pouvoir s'occuper de son enfant ". Le syndicat fait savoir à l'intéressé qu'il s'est engagé à retourner en Égypte à l'issue de sa formation et lui assure qu'il ne peut approuver le mariage ou la prolongation du séjour "car il s'agit d'affaires privées ". Le verdict est sans appel : I’Égyptien " doit repartir le 31 juillet ". Les archives ne donnent malheureusement que trop peu d'informations pour déterminer clairement les critères qui préludent à la délivrance d'un permis de séjour. De même, il est à peu près impossible d'évaluer dans quelle mesure le mariage s'apparente à une stratégie migratoire ou à un projet personnel pour les travailleurs égyptiens. On peut supposer que drames familiaux et plans d'expatriation se côtoient, sans que les autorités est-allemandes ne définissent réellement de critères transparents préalables à I'autorisation des mariages. La confrontation des archives du FDGB et de la Stasi nous permet ainsi de reconstituer à gros traits le parcours d'un Égyptien diplômé de l'École de technique agraire de Triptis en 1976, M. K. ${ }^{75}$, qui est parvenu, lui, à obtenir un permis de séjour en RDA à l'issue de sa formation. Arrivé en RDA en janvier $1974^{76}$ à I'âge de trente ans, il était prévu qu'il reparte en Égypte avec ses collègues en juin 1975. Sans que I'on sache s'il a bien quitté ou non la RDA en 1975, il est en tout cas devenu, en 1986, résident permanent à Karl-Marx Stadt et s'est marié à une Allemande de I'Est $^{77}$. Ingénieur dans une entreprise de bonification foncière, la VEB Meliorationsbau de Karl-Marx Stadt, et doctorant à I'Université technique de la ville, il a visiblement obtenu le droit d'épouser une citoyenne de RDA à l'issue de sa première formation, pourtant encadrée par le FDGB. II réside donc de manière parfaitement légale en Allemagne de I'Est. Les sources ne nous permettent pas de comprendre si, dans le cas de M.K., le mariage a été l'outil préalable à l'obtention d'un permis de séjour. Néanmoins, ce parcours individuel montre que la migration de travail des Égyptiens en RDA, bien que strictement encadrée par des conventions bilatérales, peut être le point de départ d'une stratégie d'installation hors du pays d'origine, qu'elle ait été préméditée ou non. Ainsi, bien qu'en règle générale, les " [travailleurs] formés en RDA [rentrent] [...] en Égypte ${ }^{78}$, I'installation en Allemagne orientale peut donner lieu à des recompositions imprévues et à la modification de projets professionnels, résidentiels et familiaux, ou être conçue comme une étape provisoire avant de migrer à l'Ouest. Ponctuellement, la coopération culturelle et technico-scientifique conduit donc les partenaires politiques est-allemands et égyptiens à prendre en compte les conséquences sociales de la mise en relation de leurs propres populations.

74 SAPMO-BArch, DY 34/10 542, op.cit. Note sur une conversation avec le collègue Ali Aziz, RAE, stagiaire à la VEB Strakstrom Anlagenbau d'Erfurt, Berlin, 10.2.1976.

75 Les archives du FDGB donnent le nom du stagiaire, tandis que les archives de la Stasi I'ont noirci (il reste parfaitement lisible malgré tout, par transparence). Nous avons choisi de laisser le nom de cet Égyptien dans I'anonymat.

76 SAPMO-BArch, DY 34/10 542, op.cit. Lettre adressée par M. Franke à M. Beyreuther, 10.9.1974.

77 BStU, MfS, HA II, Nr. 28103, op.cit. Lettre du lieutenant-colonel Wiegand, directeur du Département II à l'Administration de district pour la sécurité d'État de Karl-Marx Stadt, au Département des étrangers du MfS, Berlin, 29.4.1986.

78 MfAA, L 187, C 65/00, op.cit. Information über ein Treffen mit Partern des Hochschulministeriums der ARÄ, Kulturattaché, Kairo, den 28.11.79. 


\section{Conclusion}

Cet article a tenté de montrer les effets relatifs de la mise en relation des populations est-allemande et égyptienne : les circulations d'un pays à l'autre contribuent certes à diffuser le savoir-faire industriel et technique est-allemand, à façonner les représentations collectives d'autrui et à créer des réseaux relationnels étoffés, dans certaines branches de la coopération. Néanmoins, l'appropriation par l'Égypte de la " culture " est-allemande, telle que la RDA tente de la présenter à l'étranger, reste limitée à la transmission de compétences techniques et échoue en ce qui concerne la formation idéologique des partenaires égyptiens. Pour ces derniers, la migration en RDA, de même que la coopération culturelle bilatérale, restent déterminées par des facteurs socio-économiques ponctuels. De ce point de vue, les acteurs culturels est-allemands ne parviennent pas à ancrer l'idée, chez leurs homologues égyptiens, d'une culture de guerre froide partagée, sous-tendue par une lutte commune contre I' "impérialisme ". Limitée dans la durée, extrêmement faible numériquement, l'entreprise volontariste de création d'un socle culturel commun menée par Berlin-Est est assimilée par l'Égypte à une ingérence inconvenante. Elle ne conduit pas à de véritables " transferts culturels ", sauf chez une élite égyptienne politique et intellectuelle très minoritaire, qui se recrute principalement au sein des sympathisants des partis de gauche, légaux ou clandestins. Les acteurs est-allemands font preuve à cet égard d'une certaine lucidité, puisque la coopération culturelle et technicoscientifique bilatérale est perçue, dès la fin des années 1970, comme un vecteur utile pour maintenir des relations officiellement dépolitisées, mais toujours actives dans le domaine économique.

\section{Références bibliographiques}

Behrends Jan, Lindenberger Thomas und Poutrus Patrice (2003) Fremde und Fremdsein in der DDR. Zu historischen Ursachen der Fremdfeindlichkeit in Ostdeutschland, Berlin, Metropol, 376 p.

Cala-Fuentes Leonel R (2007) Kubaner im realen Paradies: Ausländer-Alltag in der DDR: eine Erinnerung, Berlin, Dietz, $176 \mathrm{p}$.

Combe Sonia (2005) La Stasi, in Chantal Brodeur et Fabien Jobard Éds., Citoyens et Délateurs. La délation peut-elle être civique?, Paris, Les Éditions Autrement, pp. 52-65.

Coumel Laurent (2001) Moscou, 1960 : la fondation de l'Université de l'Amitié des Peuples, Bulletin de I'Institut Pierre Renouvin, 12, pp. 53-71.

Dagne Haile Gabriel (2004) Das Entwicklungspolitische Engagement der DDR in Athiopien. Eine Studie auf der Basis äthiopischer Quellen, Münster, Lit Verlag, $120 \mathrm{p}$.

Dennis Mike und Weiss Karin (Eds.) (2005) Erfolg in der Nische: die Vietnamesen in der DDR und in Ostdeutschland, Münster, Lit. Verlag, 168 p.

El Kenz Ali (1995) Les ingénieurs et le pouvoir, Revue Tiers-Monde, 36 (143), pp. 565-579.

Espagne Michel (1999) Les transferts culturels franco-allemands, Paris, PUF, 286 p. 
Espagne Michel et Werner Michael (1987) La construction d'une référence culturelle allemande en France : genèse et histoire (1750-1914), Annales ESC, 42 (4), pp. 969-992.

Frank Robert (2003) Penser historiquement les relations internationales, Annuaire français de relations internationales, 4, pp. 42-65.

Hübner Peter (1998) Les ouvriers en RDA. Étude d'histoire sociale, Annales. Histoire, Sciences Sociales, 53 (1), pp. 41-68.

Katsakioris Constantin (2006) L'Union soviétique et les intellectuels africains. Internationalisme, panafricanisme et négritude pendant les années de la décolonisation, 1954-1964, Cahiers du monde russe, 47 (1), pp. 15-32.

Kott Sandrine et Faure Justine (2011) Présentation, Vingtième Siècle. Revue d'histoire, Presses de Sciences-Po, 1 (109), pp. 2-10.

Kott Sandrine, Lattard, Alain et Vincent Marie-Bénédicte (2011) Histoire de la société allemande au XXe siècle. III. La RDA, 1949-1989, Paris, La Découverte, $128 \mathrm{p}$.

Krüger-Potratz Marianne (1991) Anderssein gab es nicht. Aussländer und Minderheiten in der DDR, Münster, Waxmann, 276 p.

Laurens Henry (1987) L'URSS et l'Égypte de Nasser à Sadate, in Dominique Chevallier Éd., Renouvellement du monde arabe (1952-1982) : pensée politique et confrontations internationales, Paris, Armand Colin, pp. 47-63.

Marès Antoine (Éd.) (2007) Culture et politique étrangère des démocraties populaires, Paris, Institut d'Études Slaves, 240 p.

Moine Caroline (2010) La RDA à l'heure de la " solidarité internationale " : le festival de la jeunesse de Berlin-Est, août 1973, in Chantal Metzger Éd., La République démocratique allemande. La vitrine du socialisme et l'envers du miroir (1949-1989-2009), Bruxelles, Peter Lang, pp. 289-299.

Möller Harald (2001) Geheime Waffenlieferungen der DDR im ersten Golfkrieg an Iran und Irak, 1980-1988. Eine Dokumentation, Berlin, Verlag Dr. Köster, 104 p.

Taubert Fritz (2010) La guerre d'Algérie et la République Démocratique Allemande. Le rôle de I'" autre "Allemagne pendant les "évènements " (1954 à 1962), Dijon, Éditions universitaires de Dijon, 301 p.

Vogel Wolf-Dieter (2011) Abenteuer DDR: Kubanerinnen und Kubaner im deutschen Sozialismus, Berlin, Dietz, 184 p.

Wissa-Wassef Cérès (1972) Les relations entre l'Égypte et les deux États allemands depuis la Seconde Guerre mondiale, Politique Etrangère, 37 (5), pp. 609-638. 


\section{Amélie Regnauld}

\section{... Les limites du " remodelage socialiste " : les Égyptiens formés en RDA (1969-1989)}

La mise en place des relations diplomatiques entre la RDA et l'Égypte le 10 juillet 1969 parachève le processus de rapprochement économique et culturel entamé au milieu des années 1950. Étudiants et travailleurs égyptiens sont accueillis chaque année en RDA, au titre de la "formation populaire ", qui devient I'un des pans privilégiés de la coopération bilatérale. L'encadrement très strict de ces mobilités mène à la constitution de réseaux relationnels étoffés, qui mettent en contact scientifiques, intellectuels et activistes de gauche des deux pays. Cependant, de tels liens nourrissent des attentes divergentes du côté est-allemand et du côté égyptien. Le processus de familiarisation réciproque permis par l'immersion linguistique et culturelle est néanmoins un facteur de rapprochement des élites politiques ou artistiques, même si ces liens n'excluent pas une imbrication d'intérêts, qui ne relèvent pas tous de l'idéologie. En outre, la mise en relation des populations a des conséquences sociales, qui oscillent entre confrontation et adaptation. En analysant les ressorts de la coopération culturelle et technico-scientifique bilatérale, cet article entend montrer les limites de l'entreprise de " remodelage socialiste " menée par Berlin-Est auprès de ses interlocuteurs égyptiens.

\section{"The Limits to "Socialist Remodeling": Training Egyptians in GDR (1969-1989)}

The launching of the diplomatic relations between GDR and Egypt on 10 July 1969 brought to completion an economic and cultural rapprochement process initiated as early as the mid-50s. From then on, a cohort of Egyptians nationals students and workers alike - would travel to GDR every year to be dispensed a "popular training" that was soon to become one of the major components of the bilateral cooperation between the two countries. These - tightly supervised - mobility flows gave birth to an intense networking between scientists, intellectuals and left wing activists, with strikingly dissimilar expectations on each side. The acquaintance process sparked by this cultural and linguistic immersion nevertheless contributed in no small degree to the bringing closer of both countries' political and artistic elites. The relations thus achieved were complex and not always guided by pure ideology. And their social repercussions fluctuated from adaptation to confrontation. By analyzing the mechanisms of this cultural and techno-scientific bilateral cooperation, the present paper proposes to show the limits of East Berlin's attempted "socialist remodeling" of its Egyptian partner. 


\section{Los límites de la «remodelación socialista»: los egipcios formados en la RDA (1969-1989)}

El establecimiento de las relaciones diplomáticas entre la RDA y Egipto el 10 de julio de 1969 completó el proceso de acercamiento económico y cultural iniciado a mediados de los años 1950. Cada año, la RDA acogía a estudiantes y trabajadores egipcios a través de la llamada "formación popular», que se convirtió en uno de los sectores privilegiados de la cooperación bilateral. La consolidación de estas movilidades llevó a la constitución de relaciones y redes sólidas, poniendo en contacto a científicos, intelectuales y activistas de izquierda de los dos países. De estas relaciones entre la parte este-alemana y la egipcia surgieron también expectativas que divergían de un grupo al otro. Sin embargo, el proceso de familiarización recíproco construido a través de la inmersión lingüística y cultural fue un factor de acercamiento entre las élites políticas y artísticas existiendo incluso entre ellas intereses comunes que no solo derivaban del aspecto ideológico. Asimismo, del contacto que surge entre estas poblaciones deriva una serie de consecuencias sociales que oscilan entre la confrontación y la adaptación. Analizando los motores de la cooperación cultural y técnico-científica bilateral, este artículo pretende mostrar los límites de la puesta en marcha de la "remodelación socialista» llevada a cabo por el Berlín-Este con sus interlocutores egipcios. 